Health \& Medicine | H. Robert Guy

\title{
When the body turns against itself
}

Amyloid-based diseases

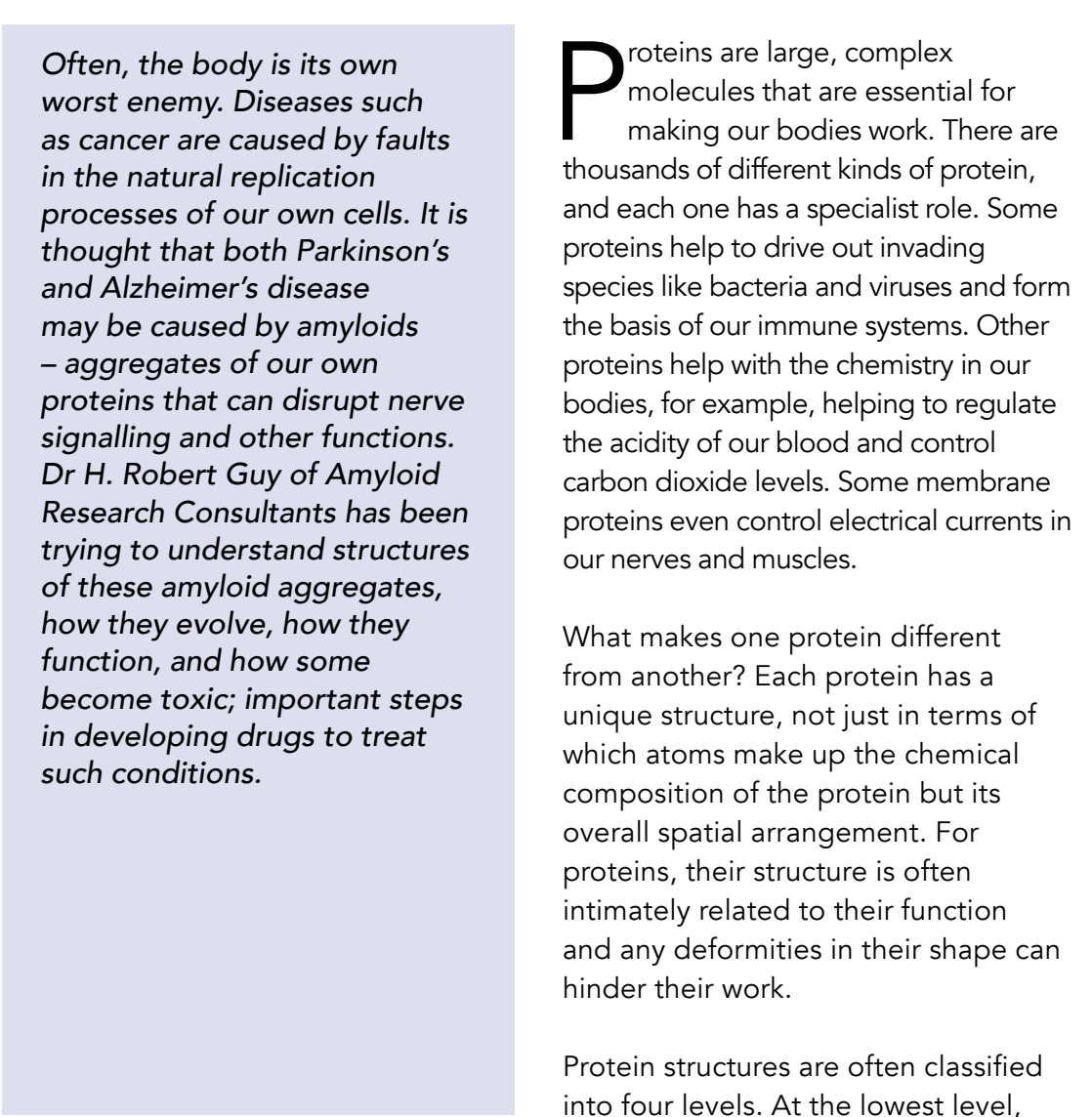

you have the primary structure which describes the chemical motifs that make up the protein. These are amino acid chains, known as polypeptide chem, that are linked together by secondary structure. The amino acid chains that make up a protein might contain over 500 amino acids, and so they often curl up and form complex shapes due to chemical interactions between different parts of the chain and the resulting structure is known as the secondary structure.

The tertiary structure of a protein is essentially its three-dimensional arrangement in space. Unlike the secondary structure, which describes localised structures in the overall protein, the tertiary structure takes into its full thape. All proteins heve ture of its full shape. All proteins have tertiany multiple polypeptide chains that act as subunits within the full structure of the protein. This gives an additional layer of structure, known as the quaternary

PRIMARY
structure SECONDARY
structure

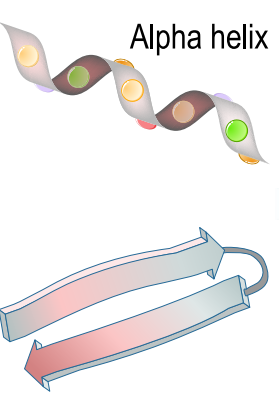

Amino acid
Beta sheet
TERTIARY
structure

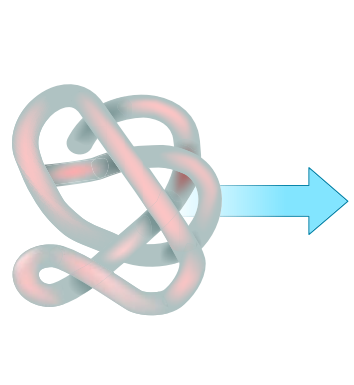

Peptide
QUATERNARY

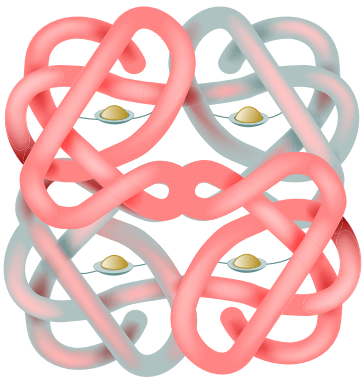

Protein structure and these subunits can or for more complex functionality. The complexity of all of these layers in shaping hundreds of chemical. structures means that sometimes things go wrong, something that Dr Robert Guy at Amyloid Research Consultants (ARC) has been deeply interested in. He has been trying to understand how the shapes of proteins might relate to their role in diseases such as Parkinson's and Alzheimer's with the hope of finding

\section{STRUCTURE MISHAPS}

Many devastating diseases including Creutzers, Parkinsons, type 2 diabetes, and some cancers are related to the presence of amyloid proteins Most a incurable or difficult to treat Amyloids usually clump together to form large waxy, fibrous structures. These fibrils are easy to identify, and portions of their three-dimensional structures have been determined experimentally.

But it is becoming increasingly clear that fibrils are not the only, or maybe even the major, cause of these diseases. What makes these amyloidbased diseases so difficult to cure is a quirk of the amyloid proteins themselves. Before forming fibrils, amyloid proteins form a large range of oligomers and protofibrils. The number of polypeptide chains within oligomers can vary from two to hundreds. As their size increases, they adopt a hugely diverse range of secondary structures. Some have sheet-like assemblies, some helices, and som are disordered.

Knowing a protein's structure and functional mechanisms are key to finding effective drugs and antibodies for treating protein-based diseases as the shape of the protein affects which chemicals can bind and alter the phall's effects. Thus, despite the challenges of trying to obtain it, many researchers have performed
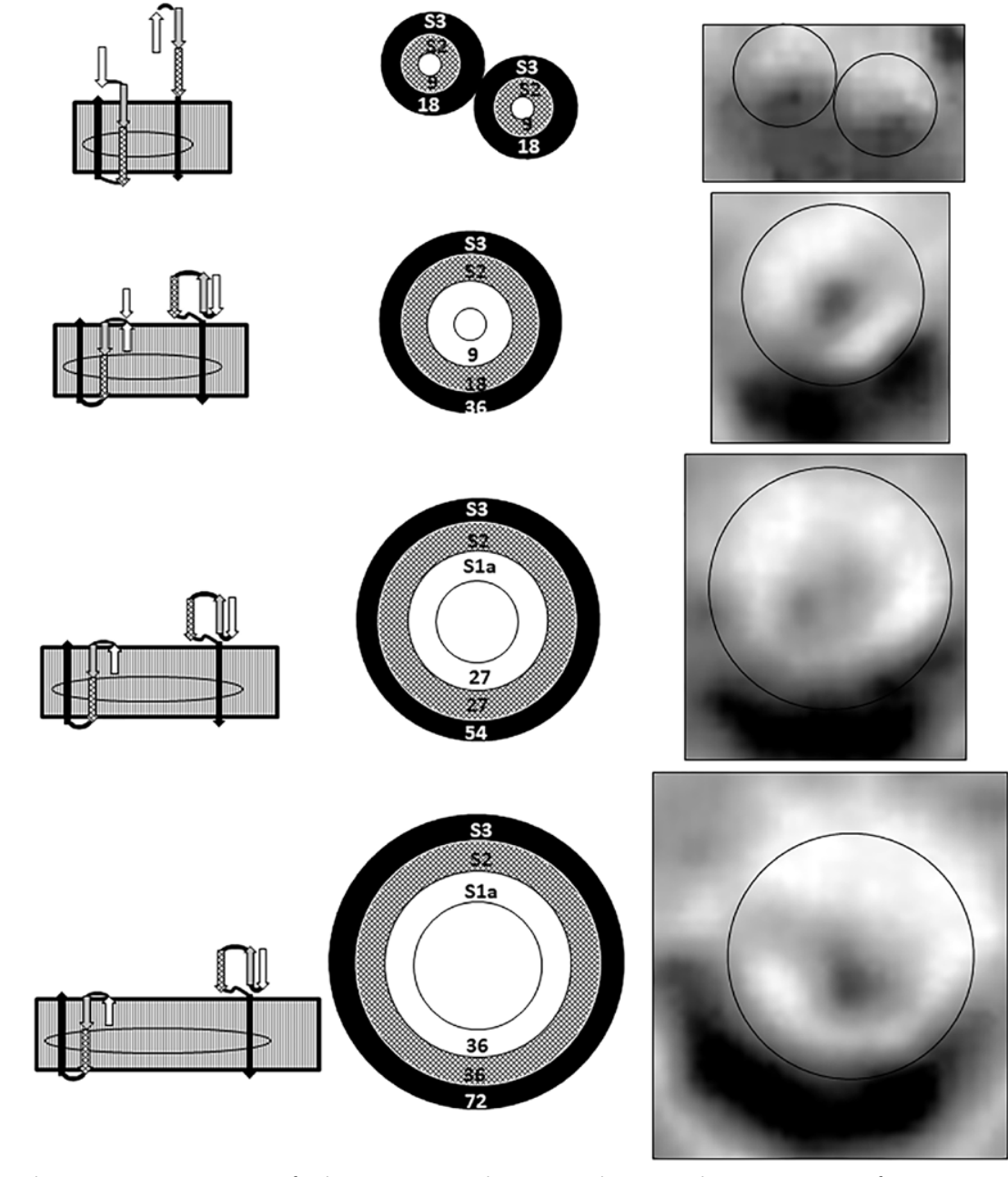

Schematic representation of subunit transmembrane topologies and
$\beta$-barrel models and microssopic images of amyloid beta chanels.

This predictability is an essential part of future drug design.

modelling to try and solve unknown to the protein structure problem protein structures.

dynamical changes. The number of polypeptides comprising amyloids Dr Guy has also been investigating an increases with time from only a few additional dimension of complexity

to thousands of fibrils. As they grow

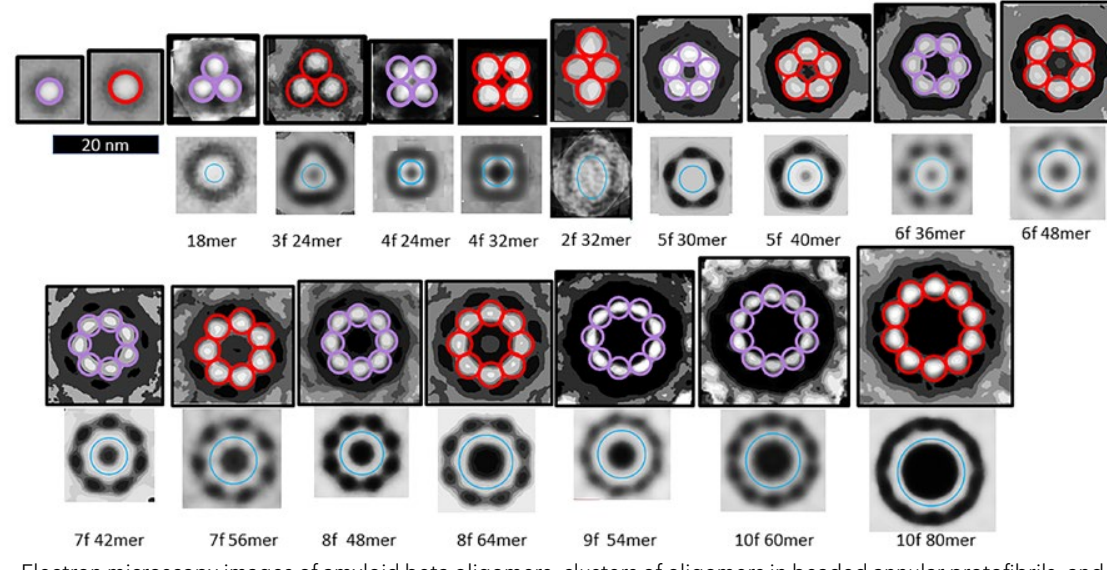

lectron microscopy images of amyloid beta oligomers, clusters of oligomers in beaded annular protofibirils, and 


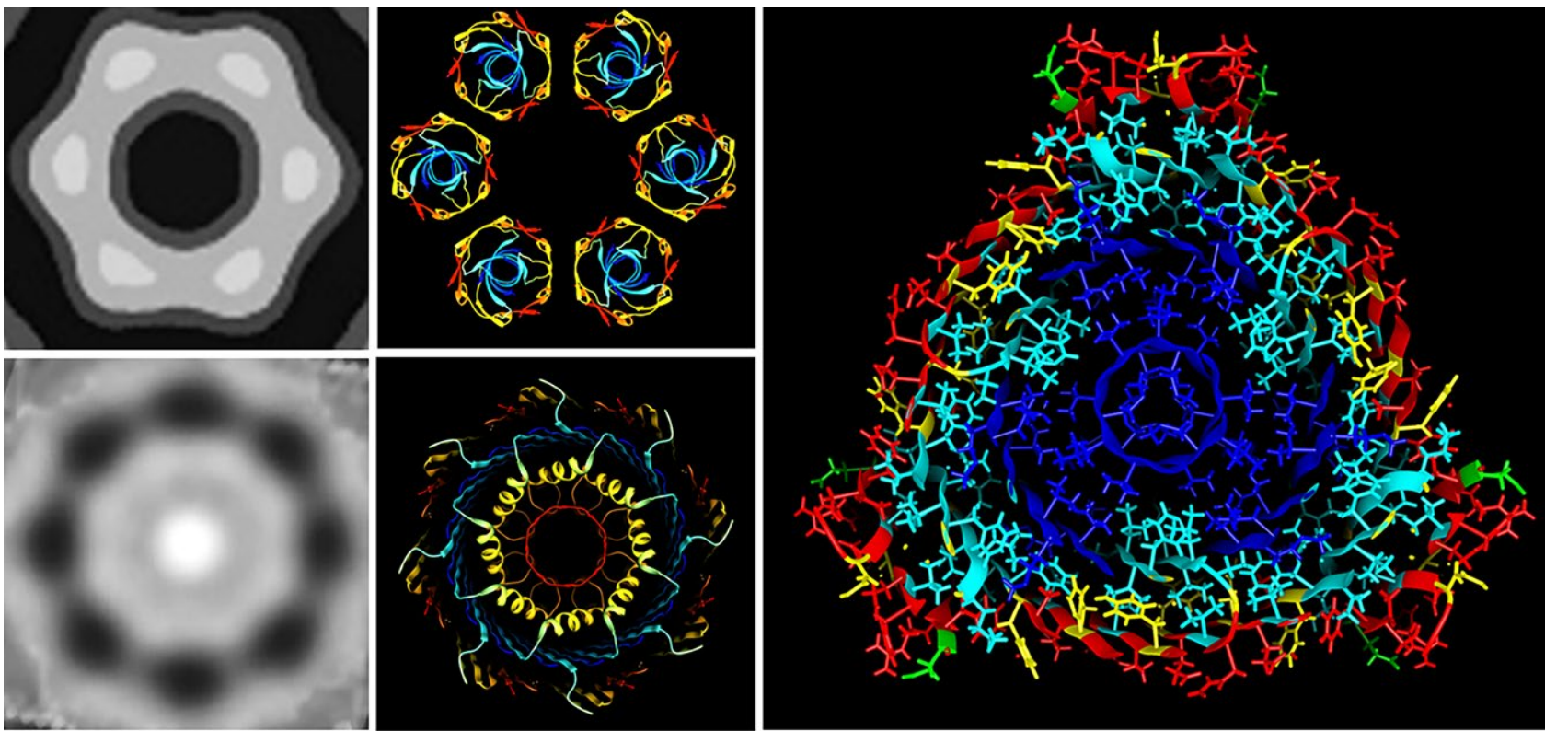

Dr Guy's models have the shapes and sizes of electron microscopy images of oligomers and annular protofibrils and consider how they evolved.

and spread to different cellular structures change dramatically. Some assemblies adopt structures vital for normal brain functions, but others deviate to toxic structures. Electrical impulses both within and between neurons are caused by the transient opening of proteins, called channels, that span the neuron's membrane. B-sheets. For smaller assemblies of some channels, they also form channels by themselves. Altering or 'shortcircuiting' the electrical currents is the last thing one would want to happen.

WIDE ANGLE

VIEW

What $\mathrm{Dr}$ Guy has been doing is trying to bring together the vast array of data available to find a hypothesis to explain the possible structure of these smaller polypeptides that are known as oligomers. Experimentally their shapeshifting ways make detailed molecular structures difficult to determine, but there is a wealth of low resolution data available on their sizes and shapes and effects on neurons plus high resoly
related fibril structures. Dr Guy has found one class of would be consistent with all of the available information for oligomers and transmembrane channels. Fibrils have multiple layers of $\beta$-sheets. The backbone chains of $\beta$-strands are stretched as much as possible. The thread-like structures of adjacent monomers bind together to form

protofibrils and transmembrane well developed, so if the number of $\beta$-strands and the $\beta$-barrel's diameter and symmetry can be determined the backbone itself is very predictable.

Hypothesis-based research is a cornerstone of contemporary science. Dr Guy's work has already made suggestions for how certain protein configurations can be stabilised.
This may help structural biologists to determine high-resolution neurobiologists to determine which and pharmaceutical companies to companies to and develop antibodies that prove these sheets can roll into cylindrical shapes to form $\beta$-barrels. Dr Guy suspects that, like fibrils, these barrels can have multiple layers. The sizes of the barrels can vary enormously and depend upon the length and

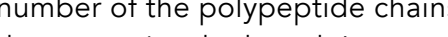
that comprise the barrel. In most conformo if this holds true conformations. If this holds true for the $\beta$-barrels it simplifies the modelling process greatly. In fact, in micrographs of amyleid ant

enges like solving the structure of small oligomers also helps develop our knowledge of the forlitities of protein

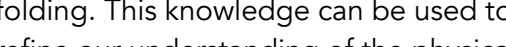
fasis of basis of certain diseases. Therefore, form will who hatin protein structures

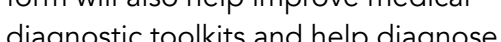
and treat people even earlier.

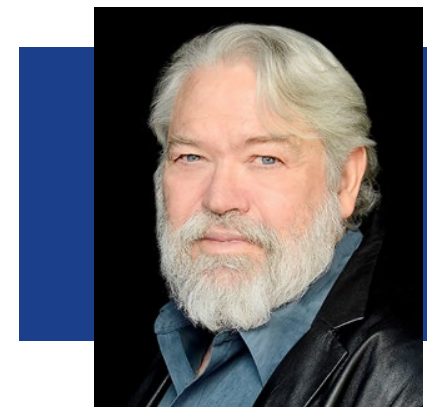

\section{Behind the Research}

Dr H. Robert Guy

\section{Research Objectives}

Robert Guy investigates amyloid-forming peptides and proteins, responsible for many neurological conditions.

\section{Detail}

Address

H. Robert Guy

A510 Thawarh Stret,

Bio

Dr Guy served as Chief of the Computational Structura

Biology Section at the National Cancer Institute. He

has published structural models of voltage-gated,

mechanosensitive, VDAC, and antimicrobial channels,

potassium transporters, and amyloid beta and synuclein oligomers, protofibrils, and transmembrane channels. He is currently CEO of Amyloid Research Consultants.

Funding

work was performed at the Nation

Cancer Institute.

Collaborators

- Stewart R. Durell

\section{References}

Durell, SR \& Guy, HR (2021). The amyloid concentric $\beta$-barrel hypothesis: models of Synuclein oligomers, annular protofibrilis, lipoproteins, and transmembrane channels. BioRxiv.

Durell, SR Kayed, R \& Guy, HR (2021). The amyloid oligomers and annular protofibrils. BioRxiv.

\section{Personal Response}

Are there any similar proteins you will be working on next?

II The next extension of our modelling could be the most significant. We are modelling how a variety of amyloids bind to AB hexamers. These amyloids 2 dide synucleins (Parkinson's disease), amylin (type and human Cystatin C (a cysteine proteinase inhibitor). Some of these interactions may be detrimental. For example, interactions between $A \beta$ and amylin, may exacerbate both Alzheimer's disease and diabetes. But other interactions appear to be beneficial; interactions the structural mechanisms could provide clues for developing new drugs and treatments for these diseases.

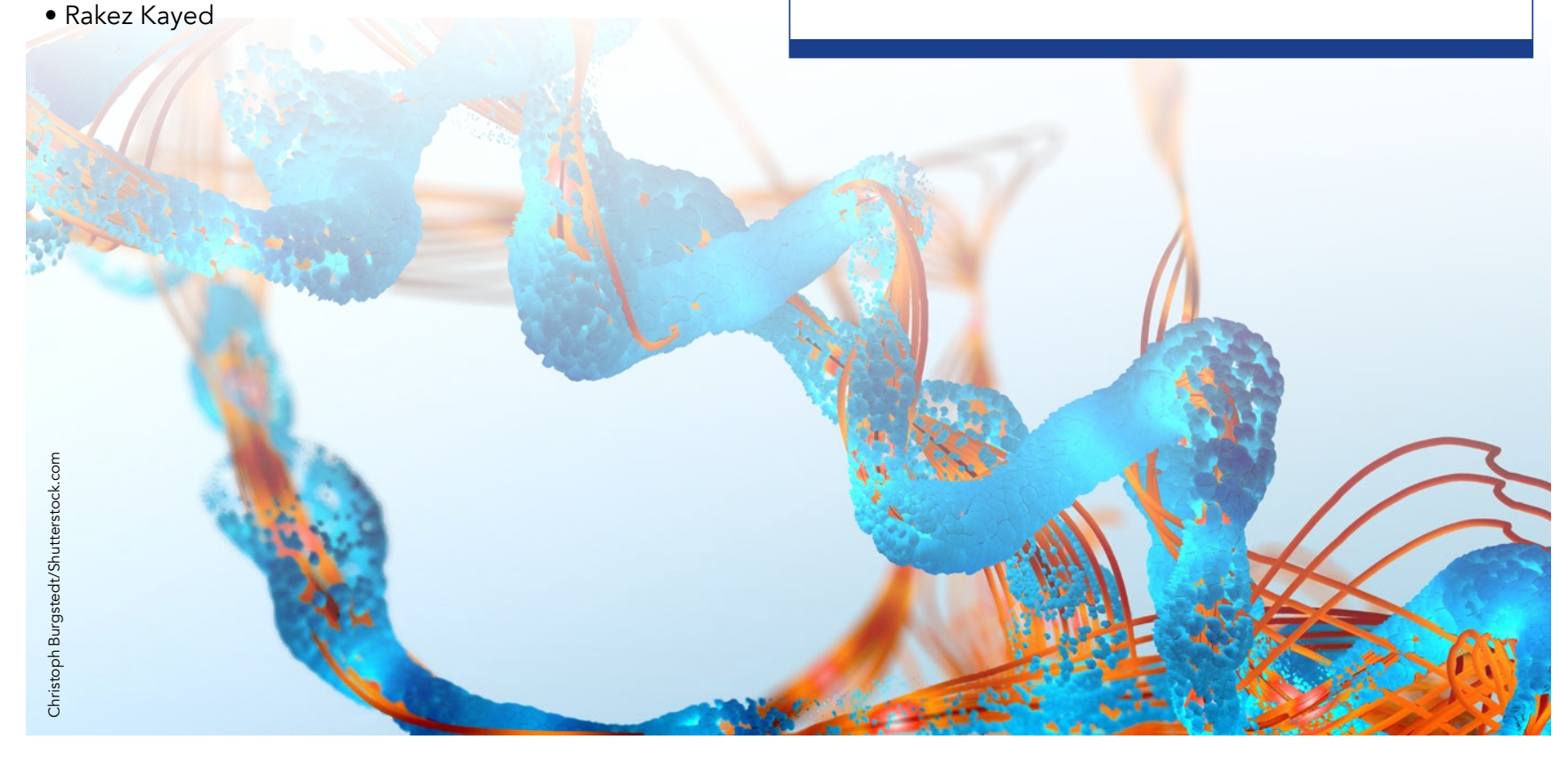

